\title{
A Learning Automata-based Heuristic Algorithm for Solving the Minimum Spanning Tree Problem in Stochastic Graphs
}

\author{
Javad Akbari Torkestani \\ Department of Computer Engineering, Islamic Azad University, Arak Branch, Arak, Iran \\ j-akbari@iau-arak.ac.ir \\ Mohammad Reza Meybodi \\ Department of Computer Engineering and IT, Amirkabir University of Technology, Tehran, Iran \\ Institute for Studies in Theoretical Physics and Mathematics (IPM), School of Computer Science, Tehran, Iran \\ mmeybodi@aut.ac.ir
}

\begin{abstract}
During the last decades, a host of efficient algorithms have been developed for solving the minimum spanning tree problem in deterministic graphs, where the weight associated with the graph edges is assumed to be fixed. Though it is clear that the edge weight varies with time in realistic applications and such an assumption is wrong, finding the minimum spanning tree of a stochastic graph has not received the attention it merits. This is due to the fact that the minimum spanning tree problem becomes incredibly hard to solve when the edge weight is assumed to be a random variable. This becomes more difficult, if we assume that the probability distribution function of the edge weight is unknown. In this paper, we propose a learning automata-based heuristic algorithm to solve the minimum spanning tree problem in stochastic graphs wherein the probability distribution function of the edge weight is unknown. The proposed algorithm taking advantage of learning automata determines the edges that must be sampled at each stage. As the presented algorithm proceeds, the sampling process is concentrated on the edges that constitute the spanning tree with the minimum expected weight. The proposed learning automata-based sampling method decreases the number of samples that need to be taken from the graph by reducing the rate of unnecessary samples. Experimental results show the superiority of the proposed algorithm over the well-known existing methods both in terms of the number of samples and the running time of algorithm.
\end{abstract}

Keyword: Learning automata, Minimum spanning tree, Stochastic graph

\section{INTRODUCTION}

Spanning tree of a connected, undirected graph is a tree-based subgraph by which all the graph vertices are connected. A minimum spanning tree (MST) of an edge-weighted graph is a spanning tree having the minimum sum of edge weights among all the spanning trees. The weight assigned to each edge of the graph represents its cost, traversal time, or length depending on the context. The minimum spanning tree is an appealing structure in the design of the communication systems that economically connect spatially dispersed elements, computer networks and other network-related problems. Minimum spanning trees also arise in more subtle applications in statistical cluster analysis $[45,46]$, data storage [47, 48,49], picture processing [50], and speech recognition [51]. Due to the tremendous growth of the communication networks, the network applications of the minimum spanning trees have 
attracted a lot of attentions during the last decades. For instance, the broadcasting problem, in which the same data must be sent to all the nodes within the network (one to all), can be simply modeled by the minimum spanning tree problem. The minimum spanning tree is also the optimal routing tree for data aggregation (all to one) in distributed environments. Besides, in some of the multicast routing protocols $[1,2]$, the minimum spanning tree is still one of the most effective and reliable methods to multicast the massages from a source node to a group of destinations. In most scenarios, the edge weight is assumed to be fixed, but such an assumption does not hold true in real world applications and the weights vary with time indeed. For example, the links in a communication network may be affected by collisions, congestions and interferences. Therefore, the MST problem is generalized toward a stochastic MST problem in which the edge weights are not constant but random variables. There have been many studies of the minimum spanning tree problem deal with the deterministic graphs and have been designed several renowned sequential algorithms such as Boruvka [3], Kruskal [4], and Prim [5] in which the MST problem can be solved in polynomial time. However, when the edge weight is allowed to be a random variable (or vary with time), the problem of finding the minimum spanning tree of the (stochastic) graph becomes incredibly difficult. This becomes more intractable, if the probability distribution function of the edge weight is assumed to be unknown.

Ishii et al. [6] proposed a method for solving the stochastic spanning tree problem in which the mentioned problem is transformed into its proxy deterministic equivalent problem and then a polynomial time algorithm presented to solve the latter problem. In this method, the probability distribution of the edge weight is assumed to be known. Ishii and Nishida [7] considered a stochastic version of the bottleneck spanning tree problem on the edges whose weights are random variables. They showed that, under reasonable restrictions, the problem can be reduced to a minimum bottleneck spanning tree problem in a deterministic case. Mohd [8] proposed a method for stochastic spanning tree problem called interval elimination. In the proposed method, like Ishii et al. [6], the problem is first transformed into a deterministic equivalent problem and then solved. Mohd also introduced several modifications to the algorithm of Ishii et al. [6]. He showed that the modified algorithm is able to obtain much better results in less time. Ishii and Matsutomi [9] presented a polynomial time algorithm to solve the problem stated in [6]. In this approach, the parameters of underling probability distribution of edge costs are assumed to be unknown, and so they are estimated by a confidence region from statistical data. In the proposed method, the problem is first transformed into a deterministic equivalent problem with a min-max type objective function and a confidence region of means and variances, since they assume that the random edge costs have normal distributions. In [52], Jain and Mamer proposed a method for estimation of the distribution of the MST weight in a stochastic network. They relaxed the condition that the random variable associated with the edge weight must be identically distributed. They obtained bounds on the distribution and the mean of the MST weight which is proved to be better than the naive bound obtained by solving the deterministic MST with expected edge weights. Alexopoulos and Jacobson's algorithm [10], which is hereafter referred to as ALJA, extended the partitioning technique considered in [40] to compute and bound specific values of the minimum spanning tree distribution in networks with independent, but not necessarily identically distributed, discrete edge weight random variables. Alexopoulos and Jacobson also proposed several methods to determine the probability that a given edge belongs to a minimum spanning tree. They demonstrated that the exact calculation of values of the minimum spanning tree distribution is NPhard.

Katagiri et al. [11] examined the case where the edge weights are fuzzy random variables. They introduced a fuzzy-based approach to model the minimum spanning tree problem in case of fuzzy random weights. Almeida et al. [12] studied the minimum spanning tree problem with fuzzy parameters and proposed an exact algorithm to solve this problem. In [13], Hutson and Shier studied several approaches to find (or to optimize) the minimum spanning tree when the edges undergo the weight changes. Repeated Prim (RP) method, cut-set (CM) method, cycle tracing (CTM) method, and multiple edge (ME) sensitivity method are the proposed approaches to find the MST of the networks in which each edge weight can assume a finite number of distinct values. To approximate the expected weight of the optimal spanning tree, Hutson and Shier used the algebraic structure to describe the relationship between different edge-weight realizations of the network. They compared different 
approaches and showed that the multiple edge sensitivity method (ME), hereafter referred to as HUSH, outperforms the others in terms of the time complexity and the size of the constructed state space. Fangguo and Huan [14] considered the problem of minimum spanning trees in uncertain networks in which the edge weights are random variables. In [14], the concept of the expected minimum spanning tree is initially defined and a model of the problem is accordingly formulated. Based on this model, a hybrid intelligent algorithm as a combination of the genetic algorithm and stochastic simulation is proposed. In order to code the corresponding spanning tree for the genetic representation, the Prüfer encoding scheme that is able to represent all possible trees is employed. Dhamdhere et al. [15] and Swamy and Shmoys [16] formulated the stochastic minimum spanning tree problem as a stochastic optimization problem and proposed some approximation approaches to solve two and multistage stochastic optimization problems.

The major problem with the above mentioned stochastic minimum spanning tree algorithms is that they are practical when the probability distribution function (PDF) of the edge weight is assumed to be known. While such an assumption does not hold true in realistic applications. In this paper, we propose a learning automata-based approximation algorithm for solving the minimum spanning tree problem in the stochastic graph, where the probability distribution function of the weight associated with the graph edge is unknown. In the proposed heuristic algorithm, by a learning automata-based sampling method, it is probabilistically decided whether an edge must be sampled or not. That is, each learning automaton which is assigned to a given graph node decides which incident edge must be sampled at each stage. In the course of the learning process, automata learn how to sample the edges along the minimum spanning tree with a higher probability. Hence, as the proposed algorithm approaches to the end, sampling process is concentrated on the edges by which the minimum spanning tree is constructed. In other words, the sampling process finally focuses on the spanning tree with the minimum expected weight. Such a probabilistic sampling method reduces the rate of unnecessary samples. To evaluate the performance of the proposed stochastic MST algorithm, the obtained results are compared with those of Alexopoulos and Jacobson [10] and Hutson and Shier [13], both in terms of the number of samples and running time of algorithm. The simulation experiments show that the proposed stochastic MST algorithm outperforms the algorithms proposed by Alexopoulos and Jacobson [10] and Hutson and Shier [13] in terms of all metrics of interest.

The rest of the paper is organized as follows. Section 2 provides a brief overview of the minimum spanning tree problems, and describes the stochastic minimum spanning tree problem. Section 3 introduces the learning automata in a nut shell. In Section 4, the proposed learning automata-based algorithm is presented. Section 5 shows the performance of the proposed algorithm through simulation experiments and comparison with the best existing methods. Section 6 concludes the paper.

\section{Minimum Spanning Tree Problem}

The Minimum Spanning Tree problem is a classical combinatorial optimization problem in graph theory. This problem is defined as to find the minimum weight spanning tree in a weighted graph. Many engineering problems such as the design of communication network, electric power system, and so on can be described by MST problem. The weight assigned to each edge of the network could represent its cost, traversal time, or length depending on the context. The minimum spanning tree problem can be generally subdivided into deterministic MST Problem and stochastic MST problem depending upon the edge weight is assumed to be fixed or a random variable. Figure 1 shows the new classification of the minimum spanning tree algorithms which is proposed in this paper.

A minimum spanning tree of a weighted, undirected graph $G$ is a spanning tree of $G$ whose edges sum to minimum weight. In other words, a minimum spanning tree is a tree formed from a subset of the edges in a given undirected graph, with two properties: first, it spans the graph, i.e., it includes every vertex in the graph, and then it is a minimum, i.e., the total weight of all the edges is as low as possible.

DEFinItion 1 Let $G\left\langle V, E>\right.$ denotes an undirected graph consisting of vertex-set $V=\left\{v_{1}, v_{2}, \ldots, v_{n}\right\}$ and edge-set $E=\left\{e_{1}, e_{2}, \ldots, e_{m}\right\} \subseteq V \times V$. Subgraph $G^{\prime}<V^{\prime}, E^{\prime}>$ of graph $G<V, E>$ is a spanning tree, if we have 


\section{J. Akbari Torkestani \& M. R. Meybodi}

1) Sub-graph $G^{\prime}$ is connected.

2) $G^{\prime}$ has the same vertex-set as $G$, i.e., $V^{\prime}=V$.

3) $\left|E^{\prime}\right|=n-1$, where $\left|E^{\prime}\right|$ denotes the cardinality of edge-set $E^{\prime}$.

Definition 2 Let $G\langle V, E, W\rangle$ denotes an edge-weighted, undirected graph, where $V=\left\{v_{1}, v_{2}, \ldots, v_{n}\right\}$ is the vertex-set, $E=\left\{e_{1}, e_{2}, \ldots, e_{m}\right\} \subseteq V \times V$ is edge-set, and $W=\left\{w_{1}, w_{2}, \ldots, w_{m}\right\}$ is the set of weights associated with the edges. Let $T=\left\{\tau_{1}, \tau_{2}, \tau_{3}, \ldots\right\}$ denotes the set of possible spanning trees of graph $G$. Let $w_{j}$ and $w_{\tau_{i}}=\sum_{\forall e_{j} \in \tau_{i}} w_{j}$ denote the weight associated with edge $e_{j} \in E$ and spanning tree $\tau_{i} \in T$. Therefore, spanning tree $\tau^{*} \in T$ is the minimum spanning tree (MST) of graph $G$, if $w_{\tau^{*}}=\min _{\forall \tau_{i} \in T}\left\{w_{\tau_{i}}\right\}$.

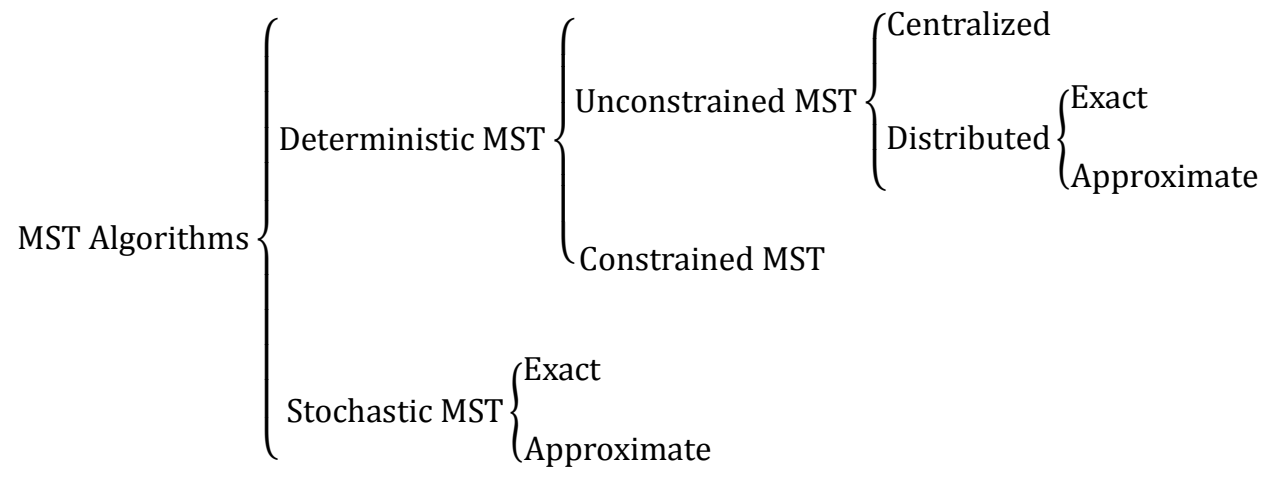

Figure 1. A new classification of the MST algorithms

\subsection{Deterministic Minimum Spanning Tree Problem}

Deterministic MST problem deals with finding the minimum spanning tree of the graph where the weight associated with the graph edge is constant. Most researches study the minimum spanning tree problem when the edge weight is constant, and so a host of deterministic algorithms are available. As shown in Figure 1, deterministic minimum spanning tree problem can be further subdivided as unconstrained and constrained MST problems. Constrained MST problem is a generalization of the MST problem in which some additional constraints must be satisfied.

Unconstrained MST algorithms are divided as centralized and distributed algorithms. Boruvka's algorithm [3], Kruskal's algorithm [4], and Prim's algorithm [5] are three well-known centralized MST algorithms. Distributed MST algorithms are further classified as exact and approximation algorithms. Gallager et al. [17], Spira [18], Dalal [19], Gafni [20], Awerbuch [21], Garay et al. [22], Elkin [23], and Kutten and Peleg [24] are representative exact solutions proposed for unconstrained MST problem. While the previous distributed algorithms deal with computing the exact MST, the next important question addressed in the literature concerns the study of distributed approximation of MST, i.e., constructing a spanning tree whose total weight is near optimal. Peleg and Rabinovich [25], Elkin [26, 27, 28], and Maleq and Pandurangan [28] proposed several approximation algorithms for finding a near optimal solution to the MST problem.

Constrained minimum spanning tree problem is a bicriteria (or multi-criteria) problem in which two (or more) parameters must be optimized. In other words, the constrained minimum spanning tree problem can be defined as a generalization of the MST problem in which some additional constraints are satisfied at the same time. Constrained minimum spanning tree problem is defined as follows.

Definition 3 Given a weighted, undirected graph $G\langle V, E, W, C\rangle$, where $V=\left\{v_{1}, v_{2}, \ldots, v_{n}\right\}$ is the vertex-set, $E=\left\{e_{1}, e_{2}, \ldots, e_{m}\right\} \subseteq V \times V$ is edge-set, $W=\left\{w_{1}, w_{2}, \ldots, w_{m}\right\}$ is the set of weights associated with the edges, and $C=\left\{c_{1}, c_{2}, \ldots, c_{m}\right\}$ denotes the (additional) constraints imposed to the edges. The constrained minimum spanning tree problem (CMSTP) can be formulated as the following optimization problem. 
$\min \sum_{\forall e_{j} \in T} w_{j}$

subject to

$\sum_{\forall e_{j} \in T} c_{j} \leq L$,

where $L$ is the imposed constraint and $T$ is the solution to the CMSTP. That is, the constrained minimum spanning tree problem is to find the spanning tree with the minimum total weight and the total constraint at most $L$.

Aggarwal et al. [29] proved that the constrained minimum spanning tree problem is a weakly NPhard problem. Representative constrained minimum spanning tree problems studied in the literature include Bounded Diameter Minimum Spanning Tree (BDMST) [30], Degree Constrained Minimum Spanning Tree (DCMST) [31], Capacitated Minimum Spanning Tree (CMST) [32], Generalized Minimum Spanning Tree (GMST) [33], Delay-Constrained Minimum Spanning Tree [34, 35], and Hop-Constrained Minimum Spanning Tree (HMST) [36]. Since the constrained minimum spanning tree problem is an NPhard problem, heuristic methods, such as tabu search [37], ant colony optimization [31], genetic algorithms [38, 39], and fuzzy-based algorithms [32], have been extensively used by the researchers for solving this complex optimization problem.

\subsection{Stochastic Minimum Spanning Tree Problem}

As mentioned above, a deterministic MST algorithm aims at finding the minimum spanning tree of the graph, where the edge weight is assumed to be fixed, while a stochastic minimum spanning tree algorithm deals with the graph edge whose weight is a random variable. In most scenarios, it is assumed that the edge weights are fixed, but this is not always true. For example, links in a communication network can malfunction or degrade as a result of congestion, accidents, weather, etc. More generally, the edges of a time-varying network can assume several states. Therefore, a deterministic graph is not able to realistically model the characteristics of such networks, and so the network topology should be modeled by a stochastic graph. As mentioned before, several algorithms have been proposed to solve the minimum spanning tree problem, where the network parameters are deterministic. However, finding the minimum spanning tree becomes considerably harder when the graph is stochastic. In what follows, we define the stochastic minimum spanning tree problem and review the stochastic MST algorithms in a nut shell.

Definition 4 A stochastic edge-weighted graph $G$ is defined by a triple $\langle V, E, W\rangle$, where $V=$ $\left\{v_{1}, v_{2}, \ldots, v_{n}\right\}$ denotes the vertex-set, $E=\left\{e_{1}, e_{2}, \ldots, e_{m}\right\} \subseteq V \times V$ denotes the edge-set, and $W=$ $\left\{w_{1}, w_{2}, \ldots, w_{m}\right\}$ denotes the set of weights associated with the edge-set such that positive random variable $w_{i}$ is the weight of edge $e_{i} \in E$.

Definition 5 Let $G\langle V, E, W\rangle$ denotes a stochastic edge-weighted graph, and $T=\left\{\tau_{1}, \tau_{2}, \tau_{3}, \ldots\right\}$ denotes the set of possible spanning trees of the stochastic graph $G\langle V, E, W\rangle$. Let $\bar{w}_{\tau_{i}}$ denotes the expected weight of spanning tree $\tau_{i}$. The stochastic MST (SMST) is defined as a stochastic spanning tree with the minimum expected weight. That is, stochastic spanning tree $\tau^{*} \in T$ is the stochastic minimum spanning tree if and only if $\bar{w}_{\tau^{*}}=\min _{\forall \tau_{i} \in T}\left\{\bar{w}_{\tau_{i}}\right\}$.

Several authors have examined network optimization problems where the edge weights are determined by independent (though not necessarily identically distributed) discrete random variables. However, in stochastic graphs, the MST problem has not received the attention it deserves. The existing stochastic minimum spanning tree algorithms are further subdivided as exact $[6,7,8,9,10]$ and approximation $[11,12,14,15,16]$ algorithms. Due to the hardness of the stochastic minimum spanning tree problem for general stochastic graphs, exact algorithms are only feasible for small graphs, while very large graphs often arise in realistic applications. Therefore, polynomial time approximation 
algorithms have been also proposed for finding a near optimal solution of the stochastic minimum spanning tree problem.

The following briefly describes two methods proposed for solving the minimum spanning tree problem in stochastic graphs with which our proposed algorithm is compared. In [40], an efficient heuristic approach was presented by Doulliez and Jamoulle for computing the probabilistic measures of the multistage systems. The proposed method is based on the iteratively partitioning the state space of the system. The proposed decomposition technique was first proposed for solving the stochastic maximum flow problem in networks with discrete arc capacities where all the flow requirements must be satisfied. Alexopoulos and Jacobson [10] enhanced the efficiency of the state space partitioning technique presented in [40] by extending and enriching its theoretical foundations. They applied the extended partitioning technique for computing the probability distribution of the weight of the minimum spanning trees in stochastic graphs with independent, but not necessarily identically distributed, discrete edge weight random variables. They proved that the exact calculation of the parameters of the probability distribution of the weight of the minimum spanning tree is known to be NP-hard. The number of iterations of the proposed algorithm is typically small for moderate size problems, however for large graphs Alexopoulos and Jacobson's algorithm may cause an intractable computational cost. Therefore, the running time of their proposed algorithm is not necessarily polynomial. The state space partitioning technique upon which the Alexopoulos and Jacobson's algorithm is based computes the probabilistic measure (the probability distribution of the weight of the stochastic MST) by dividing all the possible edge-weight realizations (the entire state space of the problem) iteratively into subsets with known contribution to the probabilistic measure and subsets with unknown contribution. This continues until no sets with unknown contribution remain to be processed. In fact, the partitioning technique is very similar to a factoring or branch-and-bound procedure, where the nodes of the search tree are the sets with unknown contribution. At any iteration, the bounds on the probabilistic measure can be computed. As the algorithm proceeds, the bounds get more tightened and finally equal to the value of the probabilistic measure.

Hutson and Shier [13] considered several approaches to solve the minimum spanning tree problem in networks in which the edge weight can assume a finite number of distinct values. In [13], the authors believe that even for small graphs the state space of the problem (i.e., the number of possible edge-weight realizations) is massive. Therefore, to alleviate the negative impacts of the huge state space, they propose a systematic way based on Hasse diagram for generating the state space which avoids generating unnecessary and repetitive states. In this method, the algebraic structure of the underlying Hasse diagram is exploited to find the relationship between the different edge-weight realizations of the stochastic network. This algebraic structure represents a graphical form of the state space in which a state is represented by a node and an edge represents a change to the next largest weight of exactly one edge. Then, a rooted spanning tree and a traversal algorithm of that tree combine to generate each state without repetition. Hutson and Shier then proposed several approaches for calculating the minimum spanning trees associated with the above constructed state space. The proposed approaches are repeated prim (RP) method, cut-set (CT) method, cycle tracing method (CTM), and multiple edge (ME) sensitivity method. They theoretically analyzed the complexities of different approaches and showed that RP and CM are always faster than CTM. Such a result is expected since RP and CM have worst case running times of order $O\left(n^{2} N\right)$ and $O(m N)$, respectively, which dominate the complexity of order $O(n m N)$ for CTM, where $n$ is the number of nodes, $m$ is the number of edges, and $N$ denotes the number of states. They also show that ME has a worst case running time of $O(m N)$. Hutson and Shier also conducted several experiments to show the performance of the proposed algorithms. The obtained results show that the multiple edge sensitivity method (ME) outperforms the others in terms of the time complexity and the size of the constructed state space. Therefore to show the superiority of our method over the proposed approaches, we compare it with ME. The main problem with the proposed approaches in [13], specifically ME method, is that to reduce the complexities of the method they take into consideration only a small subset of all the possible realizations of the stochastic network. Such a reduced state space may cause the missing of the optimal solution or even near optimal solutions. Hence, these approaches do not assure the minimum expected solution. 


\section{Learning Automata}

A learning automaton $[43,44]$ is an adaptive decision-making unit that improves its performance by learning how to choose the optimal action from a finite set of allowed actions through repeated interactions with a random environment. The action is chosen at random based on a probability distribution kept over the action-set and at each instant the given action is served as the input to the random environment. The environment responds the taken action in turn with a reinforcement signal. The action probability vector is updated based on the reinforcement feedback from the environment. The objective of a learning automaton is to find the optimal action from the action-set so that the average penalty received from the environment is minimized.

Learning automata have been found to be useful in systems where incomplete information about the environment exists [60]. Learning automata are also proved to perform well in complex, dynamic and random environments with a large amount of uncertainties. A group of learning automata can cooperate to cope with many hard-to-solve problems. To name just a few, learning automata have a wide variety of applications in combinatorial optimization problems $[53,55]$, computer networks $[54$, $56,57,58,59]$, queuing theory [61], signal processing [62], information retrieval [63], adaptive control [64], and pattern recognition [65].

The environment can be described by a triple $E \equiv\{\alpha, \beta, c\}$, where $\alpha \equiv\left\{\alpha_{1}, \alpha_{2}, \ldots, \alpha_{r}\right\}$ represents the finite set of the inputs, $\beta \equiv\left\{\beta_{1}, \beta_{2}, \ldots, \beta_{m}\right\}$ denotes the set of the values that can be taken by the reinforcement signal, and $c \equiv\left\{c_{1}, c_{2}, \ldots, c_{r}\right\}$ denotes the set of the penalty probabilities, where the element $c_{i}$ is associated with the given action $\alpha_{i}$. If the penalty probabilities are constant, the random environment is said to be a stationary random environment, and if they vary with time, the environment is called a non stationary environment. The environments depending on the nature of the reinforcement signal $\beta$ can be classified into $P$-model, $Q$-model and $S$-model. The environments in which the reinforcement signal can only take two binary values 0 and 1 are referred to as $P$-model environments. Another class of the environment allows a finite number of the values in the interval [0, 1] can be taken by the reinforcement signal. Such an environment is referred to as $Q$-model environment. In $S$-model environments, the reinforcement signal lies in the interval $[0,1]$.

Learning automata can be classified into two main families [43]: fixed structure learning automata and variable structure learning automata. Variable structure learning automata are represented by a triple $\langle\underline{\beta} \underline{\alpha}, T\rangle$, where $\underline{\beta}$ is the set of inputs, $\underline{\alpha}$ is the set of actions, and $T$ is learning algorithm. The learning algorithm is a recurrence relation which is used to modify the action probability vector. Let $\alpha_{i}(k) \in \underline{\alpha}$ and $p(k)$ denote the action selected by learning automaton and the probability vector defined over the action set at instant $k$, respectively. Let $a$ and $b$ denote the reward and penalty parameters and determine the amount of increases and decreases of the action probabilities, respectively. Let $r$ be the number of actions that can be taken by learning automaton. At each instant $k$, the action probability vector $p(k)$ is updated by the linear learning algorithm given in Equation (3), if the selected action $\alpha_{i}(k)$ is rewarded by the random environment, and it is updated as given in Equation (4) if the taken action is penalized.

$p_{j}(k+1)=\left\{\begin{array}{lr}p_{j}(k)+a\left[1-p_{j}(k)\right] & j=i \\ (1-a) p_{j}(k) & \forall j \neq i\end{array}\right.$
$p_{j}(k+1)= \begin{cases}(1-b) p_{j}(k) & j=i \\ \left(\frac{b}{r-1}\right)+(1-b) p_{j}(k) & \forall j \neq i\end{cases}$

If $a=b$, the recurrence equations (3) and (4) are called linear reward-penalty $\left(L_{R-P}\right)$ algorithm, if $a \gg b$ the given equations are called linear reward- $\epsilon$ penalty $\left(L_{R-\epsilon P}\right)$, and finally if $b=0$ they are called linear reward-Inaction $\left(L_{R-I}\right)$. In the latter case, the action probability vectors remain unchanged when the taken action is penalized by the environment. 


\section{J. Akbari Torkestani \& M. R. Meybodi}

\subsection{Variable Action Set Learning Automata}

A variable action set learning automaton is an automaton in which the number of actions available at each instant changes with time. It has been shown in [42] that a learning automaton with a changing number of actions is absolutely expedient and also $\epsilon$-optimal, when the reinforcement scheme is $L_{R-I}$. Such an automaton has a finite set of $n$ actions, $\underline{\alpha}=\left\{\alpha_{1}, \alpha_{2}, \ldots, \alpha_{n}\right\} . A=\left\{A_{1}, A_{2}, \ldots, A_{m}\right\}$ denotes the set of action subsets and $A(k) \subseteq \alpha$ is the subset of all the actions can be chosen by the learning automaton, at each instant $k$. The selection of the particular action subsets is randomly made by an external agency according to the probability distribution $\Psi(k)=\left\{\Psi_{1}(k), \Psi_{2}(k), \ldots, \Psi_{m}(k)\right\}$ defined over the possible subsets of the actions, where $\Psi_{i}(k)=\operatorname{Prob}\left[A(k)=A_{i} \mid A_{i} \in A, 1 \leq i \leq 2^{n}-1\right]$. $\hat{p}_{i}(k)=\operatorname{Prob}\left[\alpha(k)=\alpha_{i} \mid A(k), \alpha_{i} \in A(k)\right]$ is the probability of choosing action $\alpha_{i}$, conditioned on the event that the action subset $A(k)$ has already been selected and also $\alpha_{i} \in A(k)$. The scaled probability $\hat{p}_{i}(k)$ is defined as

$\hat{p}_{i}(k)=p_{i}(k) / K(k)$

where $K(k)=\sum_{\alpha_{i} \in A(k)} p_{i}(k)$ is the sum of the probabilities of the actions in subset $A(k)$, and $p_{i}(k)=\operatorname{Prob}\left[\alpha(k)=\alpha_{i}\right]$.

The procedure of choosing an action and updating the action probabilities in a variable action set learning automaton can be described as follows. Let $A(k)$ be the action subset selected at instant $k$. Before choosing an action, the probabilities of all the actions in the selected subset are scaled as defined in equation (5). The automaton then randomly selects one of its possible actions according to the scaled action probability vector $\hat{p}(k)$. Depending on the response received from the environment, the learning automaton updates its scaled action probability vector. Note that the probability of the available actions is only updated. Finally, the probability vector of the actions of the chosen subset is rescaled as $p_{i}(k+1)=\hat{p}_{i}(k+1) \cdot K(k)$, for all $\alpha_{i} \in A(k)$. The absolute expediency and $\varepsilon$-optimality of the method described above have been proved in [42].

\section{The Proposed Stochastic MST Algorithm}

As mentioned earlier, many studies have been conducted on deterministic minimum spanning tree problem, but stochastic minimum spanning tree problem has not received the attention it deserves. On the other side, due to the stochastic nature of the real world network applications, deterministic algorithms are not capable of finding the minimum spanning tree in such stochastic networks. Therefore, in this paper, we propose a learning automata-based approximation algorithm called LASMSTA (short for learning automata-based stochastic minimum spanning tree algorithm) for finding the optimal solution of the stochastic minimum spanning tree problem, where the probability distribution function of the edge weight is unknown. The deterministic case of the optimum spanning tree problem has been well studied and until now several powerful polynomial time algorithms have been proposed. But, when the edge weight vary with time, the optimum solution of the MST problem is extremely hard to find. The aim of this paper is to show the capabilities of the learning automata for solving such a difficult problem. The proposed algorithm is based on a sampling method in which at each stage a set of learning automata determines which edges must be sampled. This sampling method may result in decreasing unnecessary samples and hence decreasing the running time of algorithm.

Let $G<V, E, W\rangle$ denotes the input stochastic graph, where $V=\left\{v_{1}, v_{2}, \ldots, v_{n}\right\}$ is the vertex-set, $E=\left\{e_{1}, e_{2}, \ldots, e_{m}\right\} \subseteq V \times V$ is the edge-set, and matrix $W$ denotes the weights associated with the edgeset. In this algorithm, a network of learning automata isomorphic to the stochastic graph is initially formed by equipping each node of the graph with a $L_{R-I}$ learning automaton. The resulting network can be described by a triple $\langle\underline{A}, \underline{\alpha}, W\rangle$, where $A=\left\{A_{1}, A_{2}, \ldots, A_{n}\right\}$ denotes the set of the learning automata, $\underline{\alpha}=\left\{\underline{\alpha}_{1}, \underline{\alpha}_{2}, \ldots, \underline{\alpha}_{n}\right\}$ denotes the set of all possible actions in which $\underline{\alpha}_{i}=\left\{\alpha_{i}^{1}, \alpha_{i}^{2}, \ldots, \alpha_{i}^{r_{i}}\right\}$ defines the set of actions that can be chosen by learning automata $A_{i}$ (for each $\underline{\alpha}_{i} \in \underline{\alpha}$ ) and $r_{i}$ is the 
cardinality of action-set $\underline{\alpha}_{i}$. Edge $e_{(i, j)}$ corresponds either to the action $\alpha_{i}^{j}$ of the learning automata $A_{i}$ or to the action $\alpha_{j}^{i}$ of the learning automata $A_{j}$. That is, each learning automaton can select each of its incident edges as an action. Choosing action $\alpha_{i}^{j}$ by automaton $A_{i}$ adds edge $e_{(i, j)}$ to the minimum spanning tree. Weight $w_{i, j}$ is the weight associated with edge $e_{(i, j)}$ and assumed to be a positive random variable with an unknown probability distribution.

In the proposed algorithm, each learning automaton can be in one of two modes active and passive. All learning automata are initially set to the passive state. The proposed algorithm consists of a number of stages and at each stage one of the possible spanning trees is randomly constructed. The proposed algorithm is based on the distributed learning automata, and to explore the spanning trees, it traverses the distributed learning automata by the backtracking technique. Each stage of the LASMSTA algorithm is initiated by choosing one of the graph vertices at random. The learning automaton corresponding to the selected vertex is activated and chooses one of its actions based on its action probability vector. The edge corresponding to the selected action is added to the spanning tree which is currently being formed. The weight associated with the selected edge is added to the total weight of spanning tree as well. To avoid the loops in the tree, each passive learning automaton prunes its actionset (or scales up its action probability vector) by disabling the actions corresponding to the edges selected so far or the edges by which a cycle may be formed. Then, the learning automaton which is at the other end of the selected edge is activated. It chooses one of its actions as the previous activated automata did. The sequential activation process of learning automata (or selecting tree edges) is repeated until either a spanning tree is constructed, or no more actions can be taken by the currently active learning automaton. In the former case, the current stage is successfully completed by finding a solution to the minimum weight spanning tree problem (this occurs when the number of selected edges is greater than or equal to $(n-1)$, where $n$ denotes the cardinality of the vertex -set), and in the latter case, the proposed algorithm traces the path induced by the activated learning automata back for finding a learning automaton with available actions. The learning automaton which is found in the backtracking process is activated again. The action-set of such an automaton has to be updated by disabling its last selected action. Now, the reactivated automaton resumes the current stage by choosing one of its possible actions as described above. The learning automaton activation (or reactivation) process is continued until formation a spanning tree. The backtracking technique proposed in this paper assures that a spanning tree will be constructed at each stage of algorithm. By the backtracking technique, each learning automaton may activate more than one of its neighbors at each stage. That is, more than one action can be chosen by each learning automaton.

As mentioned earlier, the corresponding edge is added to the spanning tree, once an action is chosen by a learning automaton. The weight associated with the selected edge is also added to the total weight of the spanning tree. Since the weight associated with the graph edge is assumed to be a positive random variable, a particular spanning tree may experience a different weight at each stage. Therefore, the proposed algorithm deals with the average weight of the spanning trees rather than their weight at each stage. To do so, at the end of stage $k$, the average weight of the selected spanning tree is computed as follows:

We suppose that spanning tree $\tau_{i}$ is selected at stage $k$. The average weight of spanning tree $\tau_{i}$ until stage $k$ is computed as

$$
\bar{w}_{\tau_{i}}^{k}=\frac{1}{k_{i}} \sum_{j=1}^{k_{i}} w_{\tau_{i}}^{j}
$$

where $k_{i}$ denotes the number of times spanning tree $\tau_{i}$ is constructed until stage $k$, and $w_{\tau_{i}}^{j}$ denotes the weight of the $j^{\text {th }}$ sample of spanning tree $\tau_{i}$, which is defined as

$w_{\tau_{i}}^{j}=\sum_{\forall(s, t) \in \tau_{i}} w_{e_{(s, t)}^{j}}^{j}$

where $w_{e_{(s, t)}^{j}}^{j}$ denotes the weight of edge $e_{(s, t)}$ as a part of the $j^{\text {th }}$ sample taken from spanning tree $\tau_{i}$. 


\section{J. Akbari Torkestani \& M. R. Meybodi}

To guarantee the convergence of the proposed algorithm to the optimal solution (i.e., minimum spanning tree), the average weight of the constructed spanning tree has to be compared with the dynamic threshold, $T_{k}$, at each stage. At stage $k>1$, the dynamic threshold is calculated as

$T_{k}=\frac{1}{r} \sum_{i=1}^{r} \bar{w}_{\tau_{i}}^{k}$

where $r$ denotes the number of all spanning trees explored until stage $k$.

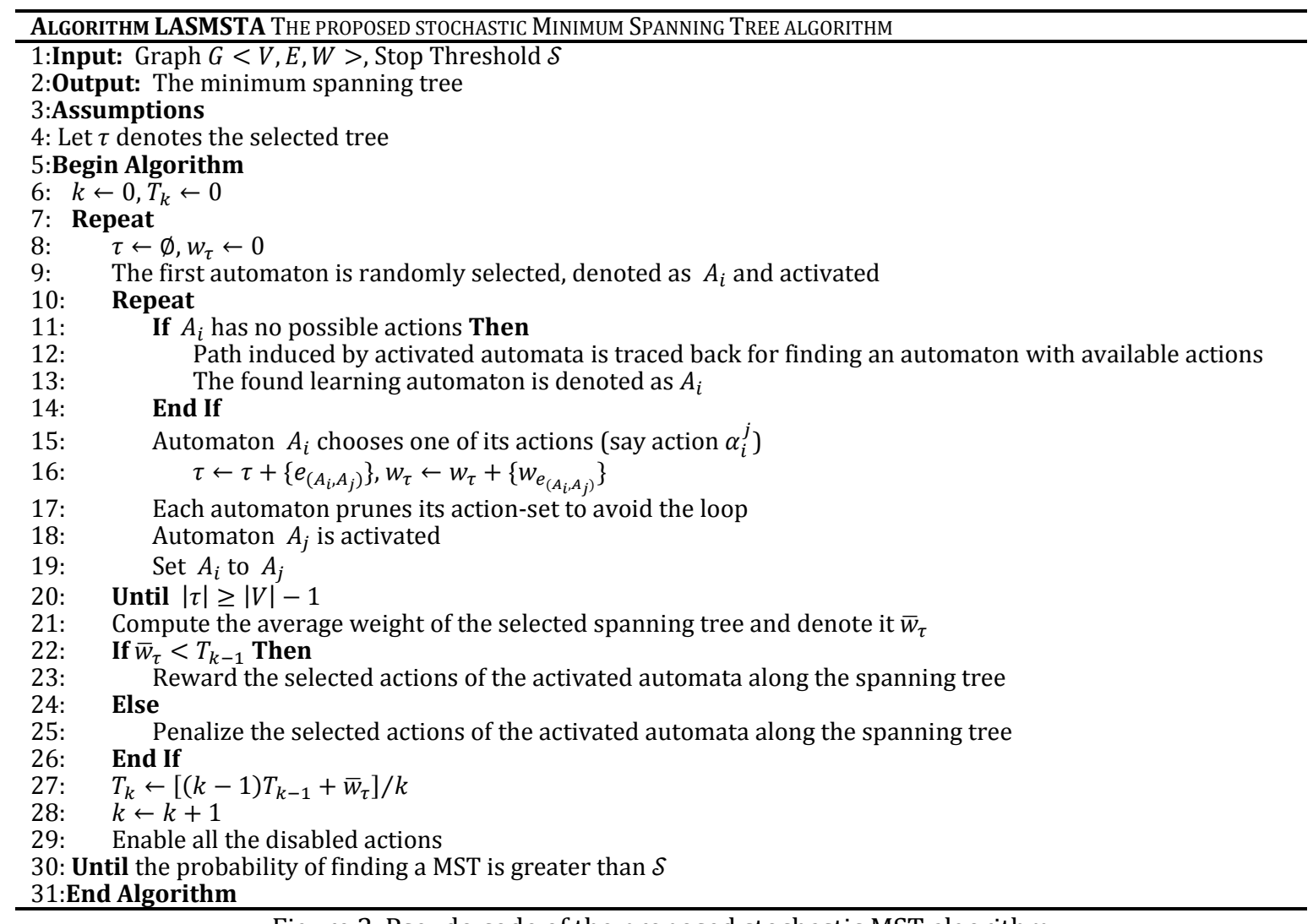

Figure 2. Pseudo code of the proposed stochastic MST algorithm

At each stage, the average weight of the selected spanning tree is compared with the dynamic threshold. All activated learning automata reward their chosen actions, if the average weight of the selected spanning tree is less than or equal to the dynamic threshold. They penalize the taken actions otherwise. Since each learning automaton updates its action probability vector by using a $L_{R-I}$ learning algorithm, the probability vectors remain unchanged when the learning automata are penalized. At the end of each stage, the disabled actions must be enabled again and the action probabilities are rescaled as described on variable action learning automata in Section 2.3. The process of constructing the spanning trees and updating the action probabilities is repeated until the choice probability of the constructed spanning tree is greater than a certain threshold $\mathcal{S}$ which is called stop threshold. The choice probability of a spanning tree is defined as the product of the probability of choosing the selected edges. The spanning tree which is selected just before the algorithm stops is the spanning tree with the minimum expected weight among all the spanning trees of the stochastic graph. Figure 2 shows the pseudo code of the proposed Stochastic MST algorithm. 


\section{EXPERIMENTAL RESULTS}

To study the performance of the proposed stochastic minimum spanning tree algorithm, we have conducted several simulation experiments on four well-known stochastic benchmark graphs borrowed from $[10,13]$. The running time of algorithm and the number of samples taken from the stochastic graph (i.e., sampling rate) are our metrics of interest. To show the outperformance of our proposed algorithm, the obtained results are compared with those of algorithms proposed by Alexopoulos and Jacobson [10] and Hutson and Shier [13]. All algorithms are tested on two sparse graphs called Alex1 and Alex 2 as well as two complete graphs with 5 and 6 vertices called $K_{5}$ and $K_{6}$, respectively. Alex1 comprises 8 nodes and 14 edges, and Alex 2 has 9 nodes and 15 edges. The discrete random variables associated with the edge weight of Alex1 and Alex2 have two and three states in mode A and B, respectively. The probability distributions of the random weights assigned to the edges of Alex 1 and Alex2 given in [41] tend toward the smaller edge weights. That is, higher probabilities are assigned to the edges with smaller weights. Such a biased distribution is more pragmatic for modeling the network dynamics than a simple uniform distribution. The other two benchmark stochastic graphs on which we tested the studied algorithms are two complete graphs $K_{5}$ and $K_{6}$ given in [13]. All stochastic MST algorithms were tested on benchmarks graphs $K_{5}$ and $K_{6}$ in two different modes and the obtained results reported in Tables 1 and 2. In the former mode denoted as E1, the distribution of the edge weight has a small variance, and in the latter mode specified as E2 the variance of the distribution associated with the edge weight is large. The random variables assigned to the edge weight of $K_{5}$ and $K_{6}$ have four and three states, respectively.

Table 1. The performance evaluation of the proposed algorithm for different learning rates

\begin{tabular}{cccc}
\hline Learning rate & RT & SR & PCR \\
\hline 0.05 & 5.9029 & 5890 & 100 \\
0.06 & 3.7890 & 4921 & 100 \\
0.07 & 2.3400 & 3764 & 100 \\
0.08 & 1.9097 & 3118 & 100 \\
0.09 & 0.9412 & 2912 & 100 \\
0.10 & 1.0350 & 2101 & 100 \\
0.15 & 0.2100 & 1623 & 98 \\
0.20 & 0.1050 & 1234 & 97 \\
0.25 & 0.0970 & 1031 & 96 \\
0.30 & 0.0783 & 989 & 93 \\
0.35 & 0.0611 & 711 & 90 \\
0.40 & 0.0510 & 590 & 88 \\
0.45 & 0.0123 & 357 & 85 \\
0.50 & 0.0098 & 210 & 81 \\
\hline
\end{tabular}

In learning automata-based algorithms, choosing the (proper) learning rate is the most challenging issue. From the learning automata theory, it is concluded that in our proposed algorithm the costs of the learning process increases and the expected weight of the spanning tree decreases (converges to the optimal one) as the learning rate decreases. That is, the solution optimality is inversely proportional to the learning rate. Such a conclusion can be also drawn from the results shown in Table 1. This property enables us to make a trade-off between the costs (running time and sampling rate) of the proposed algorithm and the optimality of the obtained solution by a proper choice of the learning rate. This means that the complexity of the proposed algorithm can be accommodated to the required optimality of the solution. To estimate the optimality of the solution, we calculate the percentage of the converged runs (PCR) to the expected weight of the minimum spanning tree for different values of learning rate. PCR is measured for 100 independent runs. To find an appropriate learning rate, we compute the running time (RT) of algorithm (in second), the total number of samples taken from the graph edges (i.e., the sampling rate of algorithm) (SR), and the percentage of the converged runs (PCR) as the learning rate of algorithm varies from 0.05 to 0.50 for Alex1. The obtained 


\section{J. Akbari Torkestani \& M. R. Meybodi}

results are shown in Table 1. Since the proposed algorithm aims at finding the minimum solution, from the obtained results it can be observed that the proposed algorithm always converges to the minimal solution with the minimum number of iterations and minimum number of samples, if the learning rate is set to 0.1 .

As discussed earlier, the simulation results given in Table 1 reveal that the running time of the proposed algorithm is inversely proportional to the learning rate. The results also show that the sampling rate and the convergence rate to the optimal solution increases as the learning rate decreases.

In learning automata-based algorithms, the convergence rate to the optimal solution is inversely proportional and the convergence speed is directly proportional to the learning rate. This is due to the fact that a learning automata-based algorithm with a small enough learning rate is capable of exploring almost all possible solutions, and so finds the best one. In fact, the costs of a learning automata-based algorithm (e.g., computational or communicational costs) increase as the learning rate decreases. As shown in Table 1, the running time of algorithm (which is the inverse of the convergence speed) increases as the learning rate becomes smaller. From Table 1, it can be seen that the sampling rate is directly proportional to the running time and increases as the learning rate increases. On the other hand, the optimality of the response (e.g., the percentage of the converged runs (PCR)) increases as the learning rate decreases. The results given in Table 1 also show that PCR increases as the learning rate decreases. This is because the number of stages of algorithm increases and so the algorithm has enough time to find the optimal (or very near to optimal) solution.

In all learning automata-based experiments conducted in this paper, the reinforcement scheme under which the action probability vector of the learning automata is updated is a linear rewardinaction $\left(L_{R-I}\right)$ algorithm with learning rate 0.1 . The stop threshold $\delta$ is set to 0.95 . This means that the proposed algorithm stops if the probability with which a spanning tree is selected becomes greater than or equal to 0.95 . Each algorithm is tested on all the above mentioned variations of the stochastic benchmark graphs Alex1, Alex2, $K_{5}$ and $K_{6}$ and the results are summarized in Tables 2 and 3 . The results reported in these tables are average over 100 runs. Table 2 shows the running time of each algorithm (in second), and Table 3 represents the total number of samples need to be taken from the graph edges (i.e., sampling rate of algorithm) by each algorithm.

\begin{tabular}{cccccc}
\multicolumn{6}{c}{ Table 2. The average running time (RT) of different algorithms (in second) } \\
\hline Graph & Vertices & Edges & ALJA & HUSH & LASMSTA \\
\hline Alex1-A & 8 & 14 & 7.412 & 3.110 & 1.035 \\
Alex2-A & 9 & 15 & 15.70 & 7.231 & 1.482 \\
Alex1-B & 8 & 14 & 19.42 & 19.45 & 1.982 \\
Alex2-B & 9 & 15 & 34.78 & 28.12 & 2.609 \\
$K_{5}$-E1 & 5 & 10 & 18.38 & 8.450 & 1.749 \\
$K_{5}$-E2 & 5 & 10 & 30.29 & 12.98 & 2.198 \\
$K_{6}$-E1 & 6 & 15 & 85.21 & 53.87 & 5.054 \\
$K_{6}$-E2 & 6 & 15 & 101.2 & 69.44 & 8.290 \\
\hline
\end{tabular}

From the numerical results reported in Table 2, it can be concluded that graphs $K_{5}$ and $K_{6}$ are more time consuming than Alex1 and Alex2. This is due to the fact that a larger number of samples must be taken from the large state space of the complete graphs (i.e., $K_{5}$ and $K_{6}$ ) for convergence to the optimal spanning tree even for smaller vertex-sets. This is also because of the probability distribution function of the random variable associated with the edge weight.

Table 2 shows the average amount of time consumed by each algorithm to solve the minimum spanning tree problem for every above mentioned stochastic benchmark graphs. Comparing the running time of ALJA, HUSH, and LASMSTA given in Table 2, it is observed that the time complexity of our proposed algorithm (LASMSTA) is significantly shorter than that of HUSH and ALJA. This is because of the fact that, unlike ALJA and HUSH, the proposed algorithm is not based on the construction of the extremely large state space of the stochastic problem. Furthermore, LASMSTA removes the nonoptimal edges (i.e., the edges that are not along the branches of the optimal spanning tree) from the sampling process for the next stages. That is, as LASMSTA proceeds the edges or branches which do not 
belong to the optimal spanning tree are pruned. As mentioned earlier, the process of constructing the problem state space is extremely time consuming for moderate size or even small stochastic graphs, and becomes an intractable problem in large networks. Therefore, ALJA and HUSH take too much time for the state space construction phase. In addition, finding the optimal tree form the huge state space requires a along time. Hence, it is expected that the running time of HUSH and ALJA will be very longer than that of LASMSTA. The obtained results given in Table 1 confirm this, showing that HUSH lags far behind LASMSTA, and ALJA takes longer time as compared with HUSH. This is because HUSH considers only a small subset of all the possible realizations of the stochastic problem state space, and avoids generating unnecessary and repetitive states. The reduced state space shortens the running time of HUSH in comparison with ALJA. On the other hand, reducing the number of states may cause the missing of the optimal state in HUSH. As a result, the probability of finding the optimal solution in HUSH is smaller than that of ALJA. Form Table 1, it can be observed that LASMSTA always converges to the optimal solution (i.e., spanning tree with the minimum expected weight) for learning rates less than 0.15, while the rate of the convergence to the optimal solution is at most 90 percent for HUSH and at most 94 percent for ALJA. Putting together the higher convergence rate and shorter running time of the proposed algorithm reveals its outperformance better.

Table 3. The average sampling rate (SR) of different algorithms

\begin{tabular}{cccccc}
\hline Graph & Vertices & Edges & ALJA & HUSH & LASMSTA \\
\hline Alex1-A & 8 & 14 & 24117 & 10760 & 2101 \\
Alex2-A & 9 & 15 & 35982 & 23412 & 2760 \\
Alex1-B & 8 & 14 & 76980 & 44902 & 3208 \\
Alex2-B & 9 & 15 & 103492 & 80981 & 3912 \\
$K_{5}$-E1 & 5 & 10 & 142981 & 53902 & 3032 \\
$K_{5}$-E2 & 5 & 10 & 192091 & 62411 & 4490 \\
$K_{6}$-E1 & 6 & 15 & 210376 & 60787 & 6210 \\
$K_{6}$-E2 & 6 & 15 & 311442 & 71192 & 6341 \\
\hline
\end{tabular}

We also conducted several simulation experiments to measure the average sampling rate (i.e., the average number of samples need to be taken from the stochastic graph) of ALJA, HUSH, and LASMSTA on different variations of the stochastic benchmark graphs Alex1, Alex2, $K_{5}$ and $K_{6}$. The obtained results are summarized in Table 3. Comparing the results given in this table, it is obvious that LASMSTA considerably outperforms the other algorithms in terms of the sampling rate. This is because the proposed learning automata-based algorithm removes the edges or branches of the graph which do not belong to the optimal spanning tree from the sampling process, and so is concentrated on the edges that construct the spanning tree with the minimum expected weight. Therefore, the proposed algorithm reduces the rate of unnecessary samples meaningfully. The results also show that the sampling rate of HUSH is very smaller in contrast with ALJA, specifically for the complete benchmark graphs. This is due to the fact that HUSH reduces the size of the problem state space by avoiding the unnecessary and repetitive states. However, sampling from the very huge state space of the stochastic problem which is constructed in ALJA and HUSH causes a mush higher sampling rate compared to LASMSTA. In addition to the lower sampling rate, LASMSTA has a higher convergence rate (to the minimal solution) in comparison with HUSH and ALJA.

\section{ConClusion}

In this paper, we first proposed a classification of the minimum spanning tree problems, and then a learning automata-based heuristic algorithm for solving the minimum spanning tree problem in a stochastic graph where the probability distribution function of the edge weight is unknown. At each stage of the proposed algorithm, the edges that must be sampled are determined by the learning automata assigned to the graph vertices. As the proposed algorithm proceeds, the sampling process is focused on the edges by which the spanning tree with the minimum expected weight is constructed. Therefore, the proposed algorithm significantly decreases the rate of unnecessary samples. To show the 


\section{J. Akbari Torkestani \& M. R. Meybodi}

performance of the proposed algorithm, we conducted several simulation experiments and compared the obtained results with those of the best existing methods. The results show the superiority of our proposed algorithm over the others in terms of the convergence rate to the optimal solution, running time of algorithm, and the sampling rate.

\section{REFERENCES}

[1] T. C. Chiang, C. H. Liu, and Y.M. Huang, "A Near-Optimal Multicast Scheme for Mobile Ad hoc Networks Using a Hybrid Genetic Algorithm,” Expert Systems with Applications, 2007, Vol. 33 pp. 734-742.

[2] G. Rodolakis, A. Laouiti, P. Jacquet, and A. M. Naimi, "Multicast Overlay Spanning Trees in Ad hoc Networks: Capacity bounds protocol design and performance evaluation," Computer Communications, 2008, Vol. 31, pp. 1400-1412.

[3] O. Boruvka, “Ojistem Problemu Minimalnım (about a certain minimal problem),” Praca Moravske Prirodovedecke Spolecnosti, 1926, Vol. 3, pp. 37-58.

[4] J. B. Kruskal, "On the Shortest Spanning Sub Tree of a Graph and the Traveling Salesman Problem," in Proceedings of the American Mathematical Society, 1956, Vol. 7, No. 1, pp. 748-750.

[5] R. C. Prim, "Shortest Connection Networks and Some Generalizations," Bell Systems Technical Journal, 1957, Vol. 36, pp. 1389-1401.

[6] H. Ishii, S. Shiode, T. Nishida, and Y. Namasuya, "Stochastic Spanning Tree Problem," Discrete Applied Mathematics, 1981, Vol. 3, pp. 263-273.

[7] H. lshii, and T. Nishida, "Stochastic Bottleneck Spanning Tree Problem,” Networks, 1983, Vol. 13, pp. 443-449.

[8] I. B. Mohd, "Interval Elimination Method for Stochastic Spanning Tree Problem," Applied Mathematics and Computation, 1994, Vol. 66, pp. 325-341.

[9] H. Ishii, and T. Matsutomi, "Confidence Regional Method of Stochastic Spanning Tree Problem," Mathematical and Computer Modeling, 1995, Vol. 22, No. 19-12, pp. 77-82.

[10] C. Alexopoulos and J. A. Jacobson, "State Space Partition Algorithms for Stochastic Systems with Applications to Minimum Spanning Trees,” Networks, 2000, Vol. 35, No. 2, pp. 118-138.

[11] H. Katagiri, E. B. Mermri, M. Sakawa and K. Kato, "A Study on Fuzzy Random Minimum Spanning Tree Problems through Possibilistic Programming and the Expectation Optimization Model," in Proceedings of the 47th IEEE International Midwest Symposium on Circuits and Systems, 2004.

[12] T. A. Almeida, A. Yamakami, and M. T. Takahashi, "An Evolutionary Approach to Solve Minimum Spanning Tree Problem with Fuzzy Parameters," in Proceedings of the International Conference on Computational Intelligence for Modelling, Control and Automation, 2005.

[13] K. R. Hutson, and D. R. Shier, "Minimum Spanning Trees in Networks with varying Edge Weights," Annals of Operations Research, Vol. 146, pp. 3-18, 2006. 
[14] H. Fangguo, and Q. Huan, "A Model and Algorithm for Minimum Spanning Tree Problems in Uncertain Networks," in Proceedings of the 3rd International Conference on Innovative Computing Information and Control (ICICIC'08), 2008.

[15] K. Dhamdhere, R. Ravi, and M. Singh, "On Two-Stage Stochastic Minimum Spanning Trees," Springer-Verlag Berlin, 2005, pp. 321-334.

[16] C. Swamy, and D. B. Shmoys, "Algorithms Column: Approximation Algorithms for 2-Stage Stochastic Optimization Problems," ACM SIGACT News, 2006, Vol. 37, No. 1, pp. 1-16.

[17] R. G. Gallager, P. A. Humblet, and P. M. Spira, "A Distributed Algorithm for Minimum Weight Spanning Trees," ACM Transaction on Programming Languages \& Systems, 1983, Vol. 5, pp. 6677.

[18] P. Spira, "Communication Complexity of Distributed Minimum Spanning Tree Algorithms," in Proceedings of the Second Berkeley Conference on Distributed Data Management and Computer Networks, 1977.

[19] Y. Dalal "Broadcast Protocols in Packet Switched Computer Networks," Technical Report 128, Department of Electrical Engineering, Stanford University, April 1977.

[20] E. Gafni, "Improvements in the Time Complexity of Two Message-Optimal Election Algorithms," in Proceedings s of the 4th Symposium on Principles of Distributed Computing (PODC), 1985, pp. 175-185.

[21] B. Awerbuch, "Optimal Distributed Algorithms for Minimum Weight Spanning Tree, Counting, Leader Election, and Related Problems," in Proceedings of the 19th ACM Symposium on Theory of Computing (STOC), 1987, pp. 230-240.

[22] J. Garay, S. Kutten, and D. Peleg, "A Sublinear Time Distributed Algorithm for Minimum-Weight Spanning Trees," SIAM Journal on Computing, 1998, Vol. 27, pp. 302-316.

[23] S. Kutten, and D. Peleg, "Fast Distributed Construction of k-Dominating Sets and Applications," Journal of Algorithms, 1998, Vol. 28, pp. 40-66.

[24] M. Elkin, "A Faster Distributed Protocol for Constructing Minimum Spanning Tree," in Proceedings s of the ACM-SIAM Symposium on Discrete Algorithms (SODA), 2004, pp. 352-361.

[25] D. Peleg and V. Rabinovich, "A Near-Tight Lower Bound on the Time Complexity of Distributed MST Construction," in Proceedings s of the 40th IEEE Symposium on Foundations of Computer Science (FOCS), 1999, pp. 253-261.

[26] M. Elkin, "Unconditional Lower Bounds on the Time-Approximation Tradeoffs for the Distributed Minimum Spanning Tree Problem," in Proceedings of the ACM Symposium on Theory of Computing (STOC), 2004, pp. 331-340.

[27] M. Elkin, "An Overview of Distributed Approximation," ACM SIGACT News Distributed Computing Column, 2004, Vol. 35 No. 4, pp. 40-57.

[28] M. Khan, and G. Pandurangan, "A Fast Distributed Approximation Algorithm for Minimum Spanning Trees," in Proceedings of the 20th International Symposium n Distributed Computing (DISC), 2006. 


\section{J. Akbari Torkestani \& M. R. Meybodi}

[29] V. Aggarwal, Y. Aneja, and K. Nair, "Minimal Spanning Tree Subject to a Side Constraint," Computer Operations Research, 1982, Vol. 9, pp. 287-296.

[30] M. Gruber, J. Hemert, and G. R. Raidl, "Neighborhood Searches for the Bounded Diameter Minimum Spanning Tree Problem Embedded in a VNS, EA and ACO," in Proceedings of Genetic and Evolutionary Computational Conference (GECCO'2006), 2006.

[31] T. N. Bui, and C. M. Zrncic, "An ant-based Algorithm for Finding Degree-constrained Minimum Spanning Tree," in Proceedings of the 8th annual conference on Genetic and evolutionary computation, 2006, pp. 11-18.

[32] T. Oncan, "Design of Capacitated Minimum Spanning Tree with Uncertain Cost and Demand Parameters," Information Sciences, Vol. 177, 2007, pp. 4354-4367.

[33] T. Oencan, J. F. Cordeau, and G. Laporte, "A Tabu Search Heuristic for the Generalized Minimum Spanning Tree Problem," European Journal of Operational Research, 2008, Vol. 191, No. 2, pp. 306-319.

[34] M. Parsa, Q. Zhu, and J. J. Garcia-Luna-Aceves, “An Iterative Algorithm for Delay-Constrained Minimum-Cost Multicasting," IEEE/ACM Transactions on Networking, 1998, Vol. 6, No. 4, pp. 461-474.

[35] H. F. Salama, D. S. Reeves, Y. Viniotis, "The Delay-constrained Minimum Spanning Tree Problem," in Proceedings of the Second IEEE Symposium on Computers and Communications, 1997, pp. 699-703.

[36] L. Gouveia, L. Simonetti, and E. Uchoa, "Modeling Hop-constrained and Diameter-constrained Minimum Spanning Tree Problems as Steiner Tree Problems over Layered Graphs," Journal of Mathematical Programming, Springer, 2009.

[37] Y. M. Sharaiha, M. Gendreau, G. Laporte, and I. H. Osman, "A Tabu Search Algorithm for the Capacitated Shortest Spanning Tree Problem,” Networks, 1998, Vol. 29, No. 3, pp. 161-171.

[38] L. Hanr, and Y. Wang, "A Novel Genetic Algorithm for Degree-Constrained Minimum Spanning Tree Problem," International Journal of Computer Science and Network Security, 2006, Vol. 6, No. 7A, pp. 50-57.

[39] M. Krishnamoorthy, and A. Ernst, "Comparison of Algorithms for the Degree Constrained Minimum Spanning Tree,” Journal of Heuristics, 2001, Vol. 7, pp. 587-611.

[40] P. Doulliez, and E. Jamoulle, “Transportation Networks with Random Arc Capacities,” RAIRO, Recherche Operationnelle Operations Research, 1972, Vol. 3, pp. 45-60.

[41] K. R. Hutson, and D. R. Shier, "Bounding Distributions for the Weight of a Minimum Spanning Tree in Stochastic Networks,” Operations Research, 2005, Vol. 53, No. 5, pp. 879-886.

[42] M. A. L. Thathachar, and B. R. Harita, "Learning Automata with Changing Number of Actions," IEEE Transactions on Systems, Man, and Cybernetics, 1987, Vol. SMG17, pp. 1095-1100.

[43] K. S. Narendra, and K. S. Thathachar, "Learning Automata: An Introduction,” New York, PrinticeHall, 1989. 
[44] S. Lakshmivarahan, and M. A. L. Thathachar, "Bounds on the Convergence Probabilities of Learning Automata," IEEE Transactions on Systems, Man, and Cybernetics, 1976, Vol. SMC-6, pp. 756-763.

[45] J. C. Gower, and G. J. S. Ross, "Minimum Spanning Trees and Single Linkage Cluster Analysis,” Journal of the Royal Statistical Society (Applied Statistics), 1969, Vol. 18, No. 1, pp. 54-64.

[46] Z. Barzily, Z. Volkovich, B. Akteke-Öztürk, G. W. Weber, “On a Minimal Spanning Tree Approach in the Cluster Validation Problem,” Informatica, 2009, Vol. 20, No. 2, pp. 187-202.

[47] S. Marchand-Maillet, Y. M. Sharaiha, "A Minimum Spanning Tree Approach to Line Image Analysis," in Proceedings of 13th International Conference on Pattern Recognition (ICPR'96), 1996, pp. 225.

[48] J. Li, S. Yang, X. Wang, X. Xue, and B. Li, “Tree-structured Data Regeneration with Network Coding in Distributed Storage Systems," in Proceedings of International Conference on Image Processing, Charleston, USA, 2009, pp. 481-484.

[49] A. N. C. Kang, R. C. T Lee, C. L. Chang and S. K. Chang, "Storage Reduction Through Minimal Spanning Trees and Spanning Forests," IEEE Transaction on Computers, 1977, Vol. C-26, pp. 425434.

[50] R. E. Osteen, and P. P. Lin, "Picture Skeletons Based on Eccentricities of Points of Minimum Spanning Trees," SIAM Journal on Computing, 1974, Vol. 3, pp. 23-40.

[51] R. L. Graham, and P. Hell “On the History of the Minimum Spanning Tree Problem,” IEEE Annals of the History of Computing, 1985, Vol. 7, No. 1, pp. 43-57.

[52] A. Jain, and J. W. Mamer, "Approximations for the Random Minimal Spanning Tree with Applications to Network Provisioning," Operations Research, 1988, Vol. 36, pp. 575-584.

[53] J. Akbari Torkestani, and M. R. Meybodi, "Learning Automata-Based Algorithms for Finding Minimum Weakly Connected Dominating Set in Stochastic Graphs," International Journal of Uncertainty, Fuzziness and Knowledge-based Systems, to appear, 2010.

[54] J. Akbari Torkestani, and M. R. Meybodi, "Mobility-based Multicast Routing Algorithm in Wireless Mobile Ad Hoc Networks: A Learning Automata Approach," Journal of Computer Communications, 2010, Vol. 33, pp. 721-735.

[55] J. Akbari Torkestani, and M. R. Meybodi, "A New Vertex Coloring Algorithm Based on Variable Action-Set Learning Automata," Journal of Computing and Informatics, 2010, Vol. 29, No. 3, pp. 1001-1020.

[56] J. Akbari Torkestani, and M. R. Meybodi, "Weighted Steiner Connected Dominating Set and its Application to Multicast Routing in Wireless MANETs," Wireless Personal Communications, Springer Publishing Company, Feb. 2010.

[57] J. Akbari Torkestani, and M. R. Meybodi, "An Efficient Cluster-based CDMA/TDMA Scheme for Wireless Mobile AD-Hoc Networks: A Learning Automata Approach," Journal of Network and Computer applications, 2010, Vol. 33, pp. 477-490. 
[58] J. Akbari Torkestani, and M. R. Meybodi, "Clustering the Wireless Ad-Hoc Networks: A Distributed Learning Automata Approach," Journal of Parallel and Distributed Computing, 2010, Vol. 70, pp. 394-405.

[59] J. Akbari Torkestani, and M. R. Meybodi, "An Intelligent Global Flooding Algorithm in Wireless Ad Hoc Networks based on Distributed Learning Automata," Journal of Computer Networks, 2010, Vol. 54, pp. 826-843.

[60] E. A. Billard, and S. Lakshmivarahan, "Learning in Multi-Level Games with Incomplete Information-Part I," IEEE Transactions on Systems, Man, and Cybernetics-Part B: Cybernetics, 1999, Vol. 19, pp. 329-339.

[61] M. R. Meybodi, "Learning Automata and Its Application to Priority Assignment in a Queuing System with Unknown Characteristics," Ph.D. thesis, Department of Electrical Engineering and Computer Science, University of Oklahoma, Norman, Oklahoma, USA, 1983.

[62] A. A. Hashim, S. Amir, and P. Mars, "Application of Learning Automata to Data Compression," New York: Plenum Press, in Adaptive and Learning Systems, K. S. Narendra ed., 1986, pp. 229234.

[63] B. J. Oommen, and E. R. Hansen, "List organizing strategies using stochastic move-to-front and stochastic move-to-rear operations," SIAM Journal of Computations, Vol. 16, pp. 705-716, Aug. 1987.

[64] C. Unsal, P. Kachroo, and J. S. Bay, "Multiple Stochastic Learning Automata for Vehicle Path Control in an Automated Highway System," IEEE Transactions on Systems, Man, and Cybernetics-Part A, 1999, Vol. 29, pp. 120-128.

[65] A. G. Barto, and P. Anandan, "Pattern-Recognizing Stochastic Learning Automata," IEEE Transactions on Systems, Man, and Cybernetics, 1985, Vol. SMC-15, pp. 360-375. 


\section{Author Biography}

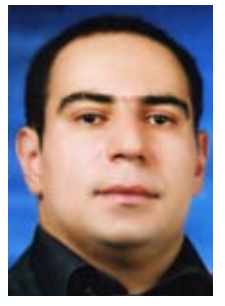

Javad Akbari Torkestani received the B.S. and M.S. degrees in Computer Engineering in Iran, in 2001 and 2004, respectively. He also received the Ph.D. degree in Computer Engineering from Science and Research University, Iran, in 2009. Currently, he is an assistant professor in Computer Engineering Department at Arak Azad University, Arak, Iran. Prior to the current position, he joined the faculty of the Computer Engineering Department at Arak Azad University as a lecturer. His research interests include wireless networks, mobile ad hoc networks, fault tolerant systems, learning systems, parallel algorithms, and soft computing.

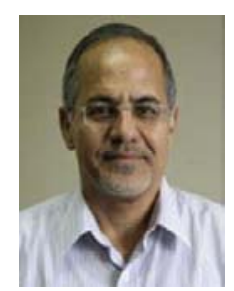

Mohammad Reza Meybodi received the B.S. and M.S. degrees in Economics from Shahid Beheshti University in Iran, in 1973 and 1977, respectively. He also received the M.S. and Ph.D. degree from Oklahoma University, USA, in 1980 and 1983, respectively in Computer Science. Currently, he is a full professor in Computer Engineering Department, Amirkabir University of Technology, Tehran, Iran. Prior to current position, he worked from 1983 to 1985 as an assistant professor at Western Michigan University, and from 1985 to 1991 as an associate professor at Ohio University, USA. His research interests include wireless networks, fault tolerant systems, learning systems, parallel algorithms, soft computing and software development. 